\title{
Parámetros Cardiovasculares y su Variación Posterior a una Atención de Urgencia Odontológica
}

\author{
Cardiovascular Parameters and their Variation after Emergency Dental Care \\ Joaquín Vázquez'; Alfio Secchi' ${ }^{1}$; Hilda Moris ${ }^{1}$; Nicolas Reyne'; Felipe Rivera²; \\ Felipe Astorga 3 ; Jorge Moreno ${ }^{4} \&$ Pablo Amorin ${ }^{4}$
} VÁZQUEZ, J.; SECCHI, A.; MORIS, H.; REYNE, N.; RIVERA, F.; ASTORGA, F.; MORENO, J. \& AMORIN, P. Parámetros
cardiovasculares y su variación posterior a una atención de urgencia odontológica. Int. J. Odontostomat 15(4):1019-1025,
2021.

RESUMEN: La atención de urgencia dental se desenvuelve en un contexto de estrés que afecta el comportamiento cardiovascular de los pacientes, expresado como cambios en Frecuencia Cardiaca y Presión Arterial. Se espera que posterior a la atención, estos parámetros varíen conforme se controla el cuadro clínico o se presenten complicaciones. Un estudio observacional retrospectivo recopiló los registros de Frecuencia Cardiaca y Presión Arterial de pacientes atendidos en la urgencia dental del Complejo Asistencial Barros Luco en dos ocasiones, estando la segunda consulta vinculada al primer ingreso, tanto como control post tratamiento o complicaciones derivadas. Datos fueron registrados en Microsoft Excel $^{\mathrm{TM}}$ y analizados en SciStat ${ }^{\mathrm{TM}}$. 466 pacientes consultaron en dos ocasiones, concentrados entre tercera y quinta década. Los mayores registros de Presión Arterial fueron reportados en controles post atención de Pericoronaritis, infecciones de espacios anatómicos faciales y en complicaciones post tratamiento por Pulpitis, TDA e Infección de espacios anatómicos faciales. En segundas consultas por control de Pulpitis, Alveolitis, TDA, Fracturas faciales y Hemorragias post exodoncia hubo disminución. Frecuencia Cardiaca fue el parámetro con mayor variación existiendo disminución en controles y complicaciones entre consultas. El papel exacto del dolor en la respuesta cardiovascular no está bien delimitado, la interpretación de las investigaciones destinadas a estudiar esto resulta difícil, pues estímulos nociceptivos son sólo uno de los activadores de la respuesta sistémica al estrés. En el postoperatorio el dolor juega un papel importante estudiado a 48 horas, más no, en mediano y largo plazo. Existen variaciones estadísticamente significativas en algunos parámetros estudiados, los cuales pueden presentar significancia clínica a considerar en pacientes hipertensos o cardiópatas. Son necesarios estudios sobre la incidencia particular que tienen los procedimientos odontológicos en la expresión de otros marcadores del estado sistémico.

PALABRAS CLAVE: agentes cardiovasculares; lesiones maxilofaciales; presión arterial; frecuencia cardiaca.

\section{INTRODUCCIÓN}

La atención de urgencias dentales conlleva en su quehacer una variedad de acciones clínicas como exodoncias, ferulizaciones, fijaciones intermaxilares, trepanaciones, entre otras. Al momento de realizar estos procedimientos, el cuerpo humano está sujeto a fenómenos de estrés fisiológico y psicológico los cuales pueden generar una respuesta en el sistema cardiovascular, la cual es posible de evidenciar al monitorizar estos pacientes (Quinto et al., 2020).
Ya se ha discutido en la literatura sobre el efecto de la ansiedad previo a procedimientos dentales de diversa índole, como tratamientos endodónticos y extracciones (Montebugoli \& Prati, 2002; Macias et al., 2013), con alzas en la presión arterial (PA) y frecuencia cardiaca (FC), de carácter transitorio a la atención, producto de la elevación de procesos simpáticos que desencadenan respuestas a nivel cardíaco y circulatorio; más no se ha revisado cómo

\footnotetext{
${ }^{1}$ Cirujano Dentista, Pasante Servicio Cirugía Maxilofacial Complejo Asistencial Barros Luco, Santiago, Chile.

${ }^{2}$ Estudiante de Odontología, Universidad de Chile, Santiago, Chile.

${ }^{3}$ Cirujano Maxilofacial, Unidad de Urgencia Dental Complejo Asistencial Barros Luco, Santiago, Chile.

${ }^{4}$ Cirujano Dentista, Unidad de Urgencia Dental Complejo Asistencial Barros Luco, Santiago, Chile.
}

Received : 21-06-2021 Accepted: 26-08-2021 
estos parámetros se ven afectados en un mediano plazo a consecuencia de procesos metabólicos que se producen en el organismo.

El manejo de una urgencia dental en gran parte de las ocasiones implica un acto quirúrgico que modifica la respuesta fisiológica de los pacientes. Las respuestas mantenidas en un organismo pueden expresarse como síntomas de hipertensión o hipotensión arterial, aumento o disminución de la frecuencia cardiaca, aumento o disminución de la temperatura corporal, problemas gastrointestinales, insomnio, tensión y mialgias (Nogareda, 1999). Aunque normalmente no se presentan estos síntomas en la atención dental regular, en un contexto de urgencia con un paciente previamente estresado a nivel fisiológico y psíquico toman relevancia tanto en el corto, mediano y largo plazo, producto de los procesos fisiológicos involucrados, que muchas veces requieren un manejo de estos parámetros previo a la atención y un seguimiento más riguroso del paciente.

En las atenciones de urgencia odontológica, la exodoncia como procedimiento ocupa un alto porcentaje, siendo más del $90 \%$ de los tratamientos, procedimiento el cual puede desencadenar una importante reacción cardiovascular en el paciente (Montebugnoli \& Prati, 2002). Aproximadamente el $10 \%$ de los pacientes sufren un alto nivel de estrés previo a la exodoncia, produciendo un aumento de la actividad simpática que puede dar como resultado una supresión vagal y una activación alfa-beta adrenérgica con posibilidad de una isquemia miocárdica por aumento del gasto cardiaco (Pando \& Expósito, 2008). Incluso en una persona habitualmente normotensa, por una situación de estrés emocional o físico, se puede presentar un pico hipertensivo que consiste en una elevación transitoria y brusca de la presión arterial (Miranda, 2009).

Múltiples estudios se han realizado estudiando el efecto de los anestésicos locales y del estrés en la atención dental, y cómo estos afectan los parámetros cardiovasculares, realizando comparaciones previas al procedimiento, al momento de la anestesia y algunos minutos posteriores a la aplicación de esta. Como también se ha revisado la utilidad de sedación en el contexto de atención dental, buscando un nivel de relajo mayor en los pacientes para así presentar menor variación de estos parámetros. Todos estos estudios realizados con pacientes sanos, sin patologías base y en un contexto de atención electiva.
El objetivo de este estudio en particular es determinar la variabilidad de la FC, PA en pacientes atendidos en un contexto de urgencia odontológica, que presentaban dolor previo al procedimiento, motivo principal de consulta en la Unidad de Emergencia del Complejo Asistencial Barros Luco, y comparar sus parámetros cardiovasculares con los obtenidos en una segunda consulta, con el cuadro doloroso resuelto o complicaciones secundarias del procedimiento realizado.

\section{MATERIAL Y MÉTODO}

Un estudio observacional retrospectivo fue realizado, recopilando la información proveniente de los Datos de Atención de Urgencia (DAU) durante un periodo de 18 meses. Este documento constituye el registro clínico de la atención recibida del paciente, presentando información de carácter crítico (nombres e identificaciones de los pacientes), estadísticos sociales (previsión, comuna de procedencia, medio de transporte al centro), y clínicos (sexo, edad, categorización de riesgo ESI, tipo de accidente, examen físico, exámenes complementarios, monitorización de FC, PAS, PAD, PAM, diagnósticos y procedimientos realizados). Los datos críticos no fueron entregados al grupo investigador, siendo los pacientes identificados por medio de un código no conocido por los investigadores, que permitiera identificar 2 consultas de la misma persona, y solo usando como motivo de estudio los datos clínicos.

La información fue ordenada y analizada por los investigadores en Microsoft Excel ${ }^{\mathrm{TM}}$, usando para este estudio los que presentaran más de una consulta al servicio de urgencia, teniendo como causal clínica del segundo ingreso, algún procedimiento o tratamiento realizado asociado al primero motivo de ingreso, siendo la segunda consulta por control de tratamiento o por complicaciones acarreadas del cuadro.

Se incluyó al análisis final todo registro que presentara una descripción íntegra del caso clínico, siendo excluidos todos los registros cuyas variables se encontraran incompletas.

La información recopilada fue organizada de acuerdo a las variables:

- Sexo en masculino o femenino

- Edad en rangos etarios por décadas 
- Categorización de riesgo según el "Emergency Severity Index" (ESI)

- Parámetros cardiovasculares (FC, PAS, PAD, PAM)

- Diagnóstico al momento del primer ingreso (Pulpitis, Traumatismo dentoalveolar o TDA, Alveolitis, Pericoronaritis, Infección de espacios anatómicos faciales, Fracturas faciales, Hemorragia post exodoncia)

- Diagnóstico al momento del segundo ingreso (Control post tratamiento, Complicación post tratamiento)

Se utilizaron estimadores puntuales de porcentaje $(\%)$, media $(\mathrm{md})$, ratio para representar las variables.

Por motivos de presentación, el valor delta entendido como la diferencia puntual entre registros, presenta valores positivos o negativos para mostrar un ascenso o descenso en el valor absoluto graficado de la variable particular.

Se calculó la significancia de relación de variables independientes con test Mann Whitney y ttest (realizadas por software SciStat ${ }^{\mathrm{TM}}$ ) con un valor de $p<0,05$ estadísticamente significativo por el tamaño de la muestra, IC = 95\%; con Hipótesis nula la variación similar.

\section{RESULTADOS}

Caracterización de la población. Durante el periodo comprendido (18 meses), ingresaron 31.182 pacientes al Servicio de Urgencia dental del Complejo Asistencial Barros Luco. De estos, 466 pacientes consultaron en por lo menos dos ocasiones producto de una patología previamente tratada en la unidad, correspondiendo a 242 mujeres y 224 hombres.

El rango etáreo de los pacientes que requirieron más de una consulta va desde los 12 a los 81 años, con promedio de 34 años, y media de 31,2 años respectivamente; se distribuyeron en grupos de los 12 a 19 años ( $n=19), 20$ a 29 años $(n=112), 30$ a 39 años $(n=134), 40$ a 49 años $(n=100), 50$ a 59 años $(n=34)$, 60 a 69 años $(n=40), 70$ a 79 años $(n=22)$, y mayores de 80 años $(n=5)$ respectivamente.

La categorización ESI al momento del ingreso fue predominantemente la ESI IV tanto en la primera como en la segunda consulta $(n=363,77,8 \% ; n=358$,
$76,8 \%)$ respectivamente, seguida por la ESI V $(n=65$, $14 \% ; n=56,12 \%)$, ESI III ( $n=34,7,3 \% ; n=46,9,9 \%)$ y ESI II ( $n=4,0,9 \% ; n=6,1,3 \%)$. No se presentaron pacientes categorizados como ESI I.

De la frecuencia de los motivos de ingreso. En la distribución de los motivos del primer ingreso, predominó la Pulpitis ( $n=331,74.2 \%)$, seguido de las Infecciones de espacios anatómicos faciales $(n=56$, $12.5 \%)$, Pericoronaritis $(n=40,8.9 \%)$, Fracturas faciales $(n=19,4.2 \%)$, Alveolitis $(n=11,2.4 \%)$, TDA $(n=6$, $1.3 \%)$ y Hemorragias post exodoncia $(n=3,0.6 \%)$.

De la tendencia de los segundos ingresos, en Pulpitis, Pericoronaritis y las Infecciones de espacios anatómicos faciales (manejados con exodoncias o tratamiento antibiótico), predominaron las por complicaciones sobre los controles post tratamiento, mientras que en los TDA, Alveolitis, Fracturas faciales y Hemorragias post exodoncias, predominaron los controles sobre las complicaciones.

la Tabla I.

Desglose de estos datos se puede apreciar en

De la variación de los parámetros cardiovasculares. Para su cálculo, se tomó la diferencia entre el promedio de las variables de manera aislada (PS, PD, PAM, FC) entre primer y segundo ingreso, siendo positivo el valor si existe un aumento del parámetro, y negativo si existió una disminución en comparación con la primera consulta. Delta nos presenta la diferencia entre los promedios de las mediciones.

De acuerdo a cada patología se presentaron las siguientes diferencias:

1. Pulpitis: sobre 331 pacientes, se presentaron más consultas por complicaciones que por control $(n=196$, $59.2 \%$ versus $n=135,40,8 \%$ ). En todos los parámetros analizados, al momento del control existió una disminución de estos, mientras que en las atenciones por complicaciones, se reportó aumento de la PS respecto a su primera consulta. FC fue el parámetro con mayor diferencia entre controles y complicaciones con $13.59 \mathrm{ppm}$ promedio de variación, única variable estadísticamente significativa.

2. Traumatismo dentoalveolar: sobre 6 pacientes, se presentaron más consultas por control que por complicaciones $(n=5,83.4 \%$ versus $n=1,16.6 \%)$. PS, PD y PAM presentaron bajas en los dos tipos de consulta, 
siendo de mayor amplitud en las complicaciones. FC no se vio afectada por las complicaciones. No se presentan datos estadísticamente significativos, lo cual podemos atribuir al bajo $\mathrm{n}$ muestral.

3. Alveolitis: sobre 11 pacientes, se presentaron más consultas por controles que por complicaciones $(n=10$, $90.9 \%$ versus $n=1,9.1 \%$ ). PS, PD, PAM y FC presentan disminución al momento de los controles, mientras que en las consultas por complicaciones existieron alzas en PS y PAM.

4. Pericoronaritis: sobre 40 pacientes, se presentaron más consultas por complicaciones que por controles $(n=18,45 \%$ versus $n=22,55 \%)$. PS y FC se presentó disminuida en los controles, mientras que en las complicaciones existió un alza de todas las variables.
5. Infección de espacios anatómicos faciales

a. Cuando se realizó extracción la primera atención: sobre 41 pacientes, se presentaron más consultas por complicaciones que por controles $(n=22,53.7 \%$ versus $n=19,46.3 \%$ ).

b. Cuando se realizó tratamiento antibiótico en la primera atención: sobre 15 pacientes, se presentaron más consultas por complicaciones que por sobre controles $(n=10,66.7 \%$ versus $n=5,33.3 \%)$.

En ambos tipos de intervenciones, PS, PD y PAM presentaron alzas al momento del control y bajas respecto al consultar con complicaciones. En ambas situaciones, se presentaron bajas de FC. Casi todas las variaciones resultan estadísticamente significativas.

Tabla I. Diferencias de valor en parámetros cardiovasculares entre consultas.

\begin{tabular}{|c|c|c|c|c|c|c|}
\hline \multirow[b]{2}{*}{$1 e r$} & \multicolumn{2}{|c|}{ rden de Ingreso } & \multicolumn{3}{|c|}{ Parámetros } & \multirow[b]{2}{*}{$\mathrm{n}$} \\
\hline & 2 do & $\begin{array}{c}\mathrm{PS} \\
(\mathrm{mmHg})\end{array}$ & $\begin{array}{c}\mathrm{PD} \\
(\mathrm{mmHg})\end{array}$ & $\begin{array}{c}\text { PAM } \\
(\mathrm{mmHg})\end{array}$ & FC (ppm) & \\
\hline \multirow{4}{*}{ Pulpitis } & Control & $-3,51$ & $-1,57$ & $-2,21$ & -15 & 135 \\
\hline & Complicación & 0,81 & $-1,98$ & $-1,05$ & $-1,41$ & 196 \\
\hline & delta & 4,32 & 0,41 & $-1,16$ & $-13,59$ & \\
\hline & $p$ & 0,054 & 0,092 & 0,067 & $<0,05$ & \\
\hline \multirow{4}{*}{ TDA } & Control & $-0,4$ & -4 & $-2,8$ & $-0,8$ & 5 \\
\hline & Complicación & -5 & $-8,3$ & -10 & 0 & 1 \\
\hline & delta & 4,6 & 4,3 & 7,2 & $-0,8$ & \\
\hline & $p$ & * & * & * & * & \\
\hline \multirow{4}{*}{ Alveolitis } & Control & $-4,7$ & $-9,8$ & $-8,1$ & $-2,4$ & 10 \\
\hline & Complicación & 17 & -5 & 2,3 & -13 & 1 \\
\hline & delta & $-21,7$ & $-4,8$ & $-10,4$ & 10,6 & \\
\hline & $p$ & * & * & * & * & \\
\hline \multirow{4}{*}{ Pericoronanitis } & Control & $-0,77$ & 2,4 & 1,37 & $-6,3$ & 18 \\
\hline & Complicación & 0,5 & 3,59 & 2,56 & $-1,63$ & 22 \\
\hline & delta & $-1,27$ & $-1,19$ & $-1,19$ & $-4,67$ & \\
\hline & $\mathrm{p}$ & 0,063 & 0,061 & 0,051 & $<0,05$ & \\
\hline \multirow{8}{*}{$\begin{array}{l}\text { Infección de } \\
\text { espacios } \\
\text { anatómicos faciales }\end{array}$} & Control post extracción & 4,78 & 2,26 & 3,1 & $-1,05$ & 19 \\
\hline & Complicación post extracción & $-8,36$ & $-3,27$ & $-4,96$ & $-8,27$ & 22 \\
\hline & delta & 13,14 & 5,53 & 8,06 & 7,22 & \\
\hline & $\mathrm{p}$ & $<0,05$ & $<0,05$ & $<0,05$ & $<0,05$ & \\
\hline & Control post tto ATB & 2 & 2,67 & 2,44 & $-7,67$ & 5 \\
\hline & Complicación post tto ATB & $-8,5$ & $-2,8$ & $-4,7$ & $-8,3$ & 10 \\
\hline & delta & 10,5 & 5,47 & 7,14 & 0,63 & \\
\hline & $p$ & $<0,05$ & $<0,05$ & $<0,05$ & 0,068 & \\
\hline \multirow{4}{*}{ Fracturas Faciales } & Control & $-3,52$ & $-7,17$ & $-5,96$ & $-9,82$ & 17 \\
\hline & Complicación & 10,5 & -3 & 1,5 & $-8,5$ & 2 \\
\hline & delta & $-14,02$ & $-4,17$ & $-7,46$ & $-1,32$ & \\
\hline & $p$ & $<0,05$ & $<0,05$ & $<0,05$ & 0,062 & \\
\hline \multirow{4}{*}{$\begin{array}{l}\text { Hemorragia post } \\
\text { exodoncia }\end{array}$} & Control & 8,5 & $-10,5$ & $-4,16$ & 0,5 & 2 \\
\hline & Complicación & 17 & -5 & 2,33 & -13 & 1 \\
\hline & delta & $-8,5$ & $-5,5$ & $-6,49$ & 13,5 & \\
\hline & $p$ & * & * & $*$ & * & \\
\hline
\end{tabular}


VÁZQUEZ, J.; SECCHI, A.; MORIS, H.; REYNE, N.; RIVERA, F.; ASTORGA, F.; MORENO, J. \& AMORIN, P. Parámetros cardiovasculares y su variación posterior a una atención de urgencia odontológica. Int. J. Odontostomat 15(4):1019-1025, 2021.

6. Fractura faciales: sobre 19 pacientes, se presentaron más consultas por controles que por complicaciones $(n=17,89.5 \%$ versus $n=2,10.5 \%)$. PS, PD, PAM y FC presentaron disminución de los parámetros de medición en los controles posteriores al primer manejo, mientras que FS y PAM presentaron alzas al comparar complicaciones en el manejo de las fracturas comparadas con el primer control.

7. Hemorragia post exodoncia: sobre 3 pacientes, se presentaron más consultas por controles que por complicaciones ( $n=2,66.7 \%$ versus $n=1,33.3 \%)$. En los controles posteriores al manejo se presentó un alza de PS y FC, y una baja de PD y PAM. Mientras que en las consultas por complicaciones presentaron alzas marcadas de PS y PAM y bajas en PD y FC. La mayor diferencia se presenta en FC, con una diferencia de 13,5 ppm promedio.

El desglose del resto de las variables se presenta en la Tabla I.

\section{DISCUSIÓN}

Cambios hemodinámicos tales como Presión arterial y Frecuencia cardiaca son parte de los parámetros necesarios a considerar durante la examinación clínica del paciente, ya que una taquicardia, bradicardia, alzas y bajas de presión pueden ser causal de complicaciones en el tratamiento odontológico, sobretodo en el contexto de un servicio de urgencia donde la atención de los pacientes puede ser impostergable (Arias et al., 2003).

El estudio presentado muestra si existen diferencias entre los parámetros cardiovasculares monitorizados en un box dental entre consultas, definiendo como causal de una segunda visita al servicio de urgencia controles post operatorios o complicaciones de procedimientos previamente realizados, en específico PS, PD, PAM y FC, siendo el primer estudio sobre este tema presentado.

A diferencia de estudios realizados del área donde se contrastan parte de estos parámetros previo a la atención, durante los procedimientos e inmediatamente posterior a este, como en los estudios de influencia de la ansiedad asociado a la anestesia, o a cambios vasculares en cirugías de implantes o extracciones (Montebugoli \& Pratti; Quinto et al.), nuestro estudio presenta los valores previo a los ingresos, tomados en el TRIAGE de la unidad de emergencia, en distintas situaciones clínicas y con un espacio de tiempo entre consultas que se extendió desde 6 horas hasta 7 días posterior a la primera intervención.

Respecto a la PAM, se presentó disminución de ésta, tanto en los controles como en las complicaciones posteriores al manejo de la Pulpitis y de los TDA, presentándose la principal disminución en la PD en ambos cuadros. También se presentó una disminución de la FC en ambas situaciones, mucho más marcada en los controles posteriores al manejo de la Pulpitis (disminución de 15 ppm, p<0.05), siendo la única variación estadísticamente significativa de las previamente estudiadas. Este resultado ha de basarse en la ya estudiada asociación del dolor con el aumento de la FC y PA. Steiman et al. (1982) ya habían presentado resultados sobre el aumento de la PA en pacientes tratados endodónticamente, reportando una disminución de este parámetro posterior a la resolución del cuadro doloroso (Steiman et al.). Incrementos de FC y PS entre 5 y $12 \mathrm{mmHg}$ ya habían sido reportados en tratamientos periodontales subgingivales al ser realizados sin anestesia y generando dolor o disconfort en el paciente (Borschukewitz et al., 1988).

Respecto de la FC, se presenta como el parámetro que más significancia estadística presenta, siendo relevante en su comparación en cuadros de Pulpitis, Pericoronaritis e Infecciones de espacios anatómicos. Importante recalcar que en todas las situaciones estudiadas se presentó una disminución de este parámetro, tanto en controles post tratamiento como en complicaciones secundarias al primer manejo, solo variando la intensidad de esta variación, siendo mucho más marcada en los controles como en las Pulpitis con disminución promedio de $15 \mathrm{ppm}$ y en las Fracturas faciales con disminución de promedio de 9.82 ppm. Estos hallazgos se condicen con la situación de control y manejo del cuadro agudo clínico que se presenta en la urgencia. Vale recordar que el sistema cardiovascular continuamente se adapta a estímulos externos e internos, donde la acción del corazón es afectada por el sistema endocrino y el sistema nervioso autónomo. La disminución de la producción de epinefrina por parte de la Médula Adrenal, situación presente en los cuadros de estrés sistémicos como el generado por dolor o por un accidente, ha de disminuir la FC de manera fisiológica, lo cual puede dar explicación a lo encontrado en este estudio (Nakamura et al., 2001). 
A nivel psicológico, la ansiedad o miedo a los procedimientos asociados a una mayor demanda fisiológica del organismo en una situación estresante puede aumentar la sensación de dolor. Este cuadro, al prolongarse, puede llevar a respuestas de resentimiento, ira, alteraciones emocionales las cuales con el pasar de los días podrían mantener o acrecentar una mayor acción del sistema cardiovascular, generando un aumento de la PA si no se ha controlado el cuadro doloroso o que hace consultar al paciente al servicio de urgencia, como en las complicaciones post tratamiento (Pando \& Expósito).

Fisiológicamente hay una respuesta sistémica y una local, con cambios inflamatorios, autonómicos, endocrinos e inmunológicos. El papel exacto del dolor en esta respuesta no está bien delimitado, al no poder separarse los efectos del dolor per se de aquellos producidos por la desnutrición tisular. La interpretación de las investigaciones destinadas a estudiar este problema también es difícil, pues los estímulos nociceptivos son sólo uno de los activadores de la respuesta sistémica al estrés, y casi siempre otros de estos activadores acompañan al dolor. En el postoperatorio, el dolor juega un papel importante, especialmente en las primeras 48 horas.

Hemos podido apreciar que los procedimientos odontológicos generan variaciones de los parámetros cardiovasculares, los cuales pueden ser inocuos en los pacientes ni presentar un riesgo a nivel cardiovascular, pero vale la pena mantener precauciones respecto al manejo de las situaciones de emergencia, puesto que pacientes cardiópatas o hipertensos han de poder verse afectados en el mediano plazo producto de alzas de PA como en crisis hipertensivas o disminuciones marcadas de FC producto del manejo realizado en el contexto de urgencia (Aranzazu et al., 2014).

\section{CONCLUSIONES}

Monitorización constante y seguimiento de los parámetros cardiovasculares resultan importantes entregando información sobre el control del estado sistémico del paciente, el cual ante la presencia de alguna patología o de complicaciones posteriores a procedimientos, puede presentar alzas de Presión arterial o de Frecuencia cardiaca, factores relevantes a considerar en pacientes con Hipertensión arterial o patologías coronarias.
El manejo del dolor y del estrés fisiológico y psicológico, además de constituir motivos humanitarios, pueden resultar en la menor incidencia de complicaciones cardiovasculares como lo pueden ser trombosis venosas profundas o crisis hipertensivas. Resulta relevante investigar cómo la modulación del dolor posterior a procedimientos dentales ha de influir en el estado sistémico del paciente más allá del post operatorio inmediato.

Queda a futuro como tarea el estudiar variaciones de otros parámetros sistémicos y cómo se afectan en el mediano y largo plazo, como glicemia, saturación de oxígeno, los cuales no fueron considerados producto de limitaciones operacionales del servicio.

VÁZQUEZ, J.; SECCHI, A.; MORIS, H.; REYNE, N.; RIVERA, F.; ASTORGA, F.; MORENO, J. \& AMORIN, P. Cardiovascular parameters and their variation after emergency dental care. Int. J. Morphol., 39(6):1019-1025, 2021.

ABSTRACT: Emergency dental care develops in a context of stress that affects the cardiovascular behavior of patients, expressed as changes in heart rate and blood pressure. After care, these parameters are expected to vary as the clinical picture is controlled or complications occur. A retrospective observational study compiled the heart rate and blood pressure records of patients seen in the dental emergency of the Barros Luco Care Complex on two occasions, the second consultation being linked to the first admission, as well as post-treatment control or derived complications . Data was recorded in Microsoft Excel ${ }^{\mathrm{TM}}$ and analyzed in SciStat TM. 466 patients consulted on two occasions, concentrated between the third and fifth decade. Higher Blood Pressure records were reported in post-care controls for Pericoronitis, infections of facial anatomical spaces and in post-treatment complications due to Pulpitis, ADD and Infection of facial anatomical spaces. In second consultations for control of Pulpitis, Alveolitis, ADT, facial fractures and postextraction hemorrhages there was a decrease. Heart rate was the parameter with the greatest variation, with a decrease in controls and complications between consultations. The exact role of pain in the cardiovascular response is not well defined, the interpretation of the research aimed at studying this is difficult, since nociceptive stimuli are only one of the activators of the systemic response to stress. In the postoperative period, pain plays an important role, studied at 48 hours, but not in the medium and long term. There are statistically significant variations in some parameters studied which may present clinical significance to be considered in hypertensive or heart disease patients. Studies are needed on the particular incidence that dental procedures have on the expression of other markers of the systemic state.

KEY WORDS: Cardiovascular agents; Maxillofacial injuries; Blood pressure; Heart Rate. 
VÁZQUEZ, J.; SECCHI, A.; MORIS, H.; REYNE, N.; RIVERA, F.; ASTORGA, F.; MORENO, J. \& AMORIN, P. Parámetros cardiovasculares y su variación posterior a una atención de urgencia odontológica. Int. J. Odontostomat 15(4):1019-1025, 2021.

\section{REFERENCIAS BIBLIOGRÁFICAS}

Aranzazu, M.; Delgado, J. \& Pieschacón, G. Variaciones de riesgo en valores de tensión arterial en pacientes hipertensos durante procedimientos odontológicos. Rev. Univ. Ind. Santander, 46(2):110, 2014.

Arias, B.; Romero, M.; Hita, C.; Bravo, M. \& Vallecillo, M. Seguimiento de la frecuencia cardiaca y la presión parcial de oxígeno durante la cirugía bucal. Av. Odontoestomatol., 19(2):1-6, 2003.

Borschukewitz, R.; Wetzel, W. \& Wittmann, L. Zusammenhang zwischen psychischem Angsterleben und Veränderungen der Pulsfrequenz während der zahnäztlichen Behandelung. Dtsch. Zahnartzl. Z., 43:1012-8, 1988.

Macias, D., Parra, R., Luna, C., Guerrero, F. \& Cornejo, M. Efecto del tratamiento endodóntico en los valores de la presión arterial en pacientes hipertensos. Rev. ADM, 70(1):30-4, 2013.

Miranda, A. Hipertensión Arterial, la Enfermedad Silenciosa. Revista Mercado, Editorial Coyuntura S. A., 2009.

Montebugoli, L. \& Prati, C. Circulatory dynamics during dental extractions in normal, cardiac and transplant patients. J. Dent. Assoc., 133(4):468-72, 2002.

Nakamura, Y.; Matsumura, K.; Miura, K.; Kurokawa, H.; Abe, I. \& Takata, Y. Cardiovascular and sympathetic responses to dental surgery with local anesthesia. Hypertens. Res., 24(3):209-14, 2001.

Nogareda, C. Fisiología del estrés. Instituto Nacional de Seguridad e Higiene en el Trabajo. Madrid, Ministerio de Salud y Asuntos Sociales, 1999.

Pando, P. \& Expósito, M. Exodoncia dental y riesgo cardiovascular. Variabilidad de las constantes hemodinámicas durante la exodoncia dental. Cient. Dent., 5(3):175-81, 2008.

Quinto, A.; Rodríguez, L.; Grados, S. \& Castro, Y. Variaciones de la frecuencia cardíaca, temperatura corporal y presión arterial en pacientes sometidos a cirugía de implantes dentales. Odontoestomatología, 23(36):1-9, 2020.

Steiman, H.; Patterson, S.; Newton, C.; Triup, P. \& Zipes, D. Cardiovascular changes during nonsurgical endodontics. J. Endod., 8:497-501, 1982.

\author{
Dirección para correspondencia: \\ Joaquín A. Vázquez D. \\ Hospital Barros Luco Trudeau \\ Gran Avenida José Miguel Carrera 3204 \\ San Miguel \\ Santiago \\ CHILE
}

E-mail: joaquin.vazquez.d@gmail.com 